\title{
A Duplexer Design Technology Based on Co-simulation of HFSS and ADS
}

\author{
Xiong Rong \\ Chengdu University of Information Technology \\ Institute of Communication Engineering \\ Chengdu, China
}

\author{
Yang Huan \\ Chengdu University of Information Technology \\ Institute of Communication Engineering \\ Chengdu, China
}

\begin{abstract}
A miniaturized duplexer with high isolation is designed in this paper. The coupling coefficient of branch filter is solved by Generalized Chebyshev function, and the circuit model is simulated and optimized by MATLAB and ADS. Finally, the physical dimensions are extracted by HFSS, and the simulation model of combiner is established and optimized. The experimental results show that the loss of the miniaturized combiner is less than $1.5 \mathrm{~dB}$, and the out of band rejection is greater than 40dB @ $1550-1580 \mathrm{MHz} \& 1600-$ $1625 \mathrm{MHz}$.The simulation results verify the rationality and feasibility of the design.
\end{abstract}

Keywords: Combiner; Common cavity; Coupling matrix; Model simulation

\section{INTRODUCTION}

With the rapid development of mobile communications, the demand for a compact and highly selective combiner is increasing. Combining two or more circuit system signals into one circuit not only saves material, but also eliminates the need to switch antennas during signal transmission. Compared with the traditional combiner, the current cavity combiner has many advantages, such as low insertion loss, high out-of-band suppression and large power capacity, which are widely used in broadcasting, radar and satellite communication systems [13]. Miniaturization of multi - frequency combiner has become one of the important development directions of combiner development [4].

The combiner is composed of multiple signals, so in order to ensure the communication quality of the system and prevent mutual interference between the systems, the separation of the combiner must have a high requirement. Therefore, how to design a compact and highly isolated combiner is always a problem studied by designers [5].

The current combiner is composed of two filter elements with multiple cross-coupling designs and a common cavity at the combiner end. A three-port combiner with operating frequencies ranging from $1550-1580 \mathrm{MHz}$ to $1600-1625 \mathrm{MHz}$ is designed according to this structure. In this thesis, Chebyshev filter design is adopted to obtain the coupling coefficient of two-pass filter and the number of order and zero of single-pass filter [6]. Finally, the simulation test of this combiner is carried out, and the dimensions of the combiner are reduced while the parameters of the combiner are guaranteed. This design not only makes the circuit breaker have high isolation degree, but also realizes the design structure of the combiner miniaturization to adapt to different scene requirements, which has a good application prospect.

\section{RELATED WORK}

A miniaturized duplexer method based on ads and HFSS cosimulation is designed. In the design process, in order to ensure that each channel can achieve the corresponding indicators, the coupling coefficients of the two channels are simulated in ads. Then, the coupling coefficients of each path are transformed into corresponding physical dimensions in HFSS. Finally, the performance of the diplexer model is compared with the requirements, and it is found that it meets the design requirements. This paper mainly includes the following contents:

- The S-parameter pre simulation is carried out by MATLAB software, and two independent coupling coefficients are obtained;

- The circuit model is simulated in ads with the coupling coefficient, and the external $\mathrm{Q}$ value and topology are obtained;

- The obtained structure and parameters are transformed into specific physical dimensions in HFSS, and modeling and simulation optimization are carried out

Finally, the $S$ parameters of the optimized diplexer are compared with the expected indexes.

\section{THE CIRCUIT MODEL OF THE COMBINER}

The present paper designs a three-port combiner with two channels consisting of a Bandpass filter with cross-coupling design. The main indexes are shown in Table 1.

Table 1 Design indexes of combiner

\begin{tabular}{ccc}
\hline Parameter & GPS & L $_{\text {short message }}$ \\
\hline Frequency Range/MHz & $1550-1580$ & $1600-1625$ \\
Band-width/MHz & 30 & 25 \\
IL at BW/dB & $\leq 1.2$ & $\leq 1.5$ \\
Return loss at BW/dB & $\leq-20$ & $\leq-20$ \\
Isolation/dB & $\geq 40$ & $\geq 40$
\end{tabular}

In order to minimize the attenuation in the Passband, Chebyshev filter is designed according to the formula:

$$
\begin{gathered}
k_{i, j}^{k}=B_{n} \times M_{K}(i, j) \\
Q_{e}^{k}=1 /\left\{B_{n}\left[M_{k}\left(n_{P k}+1, n_{P k}+2\right)\right]^{2}\right\} \\
B_{n}=B / f_{0} \\
f_{r i s, i}^{k}=f_{0}\left[\sqrt{1+\left(\frac{B_{n} M_{i, i}^{k}}{2}\right)^{2}}-\frac{B_{n} M_{i, i}^{k}}{2}\right]
\end{gathered}
$$




$$
\begin{array}{r}
k_{0,1}^{k}=B \times\left(M_{k}(1,2) / \sqrt{c_{0}}\right) \\
Q_{e}=c_{0} / B_{n} \\
f_{r i s, 0}=f_{0}\left[\sqrt{1+\left(\frac{B_{n} b_{0}}{2}\right)^{2}}-\frac{B_{n} b_{0}}{2}\right]
\end{array}
$$

Where, equations (1) - (4) are applied to non-common cavities, and equations (5) - (7) are applied to common cavities. $k_{i, j}^{k}$ is the internal coupling coefficient of the filter, $Q_{e}^{k}$ is the $\mathrm{Q}$ value of filter, $f_{r i s, i}^{k}$ is the resonant frequency of the resonator, $B$ is the filter bandwidth, $B_{n}$ is relative bandwidth, $M_{k}$ is the coupling matrix, $b_{0}$ is the normalized admittance of the common cavity, $c_{0}$ is normalized capacitance, $f_{0}$ is the center frequency, $n_{P K}$ is the order of the $K$-th filter[7].

According to the design theory of coupled resonator Bandpass filter and the above formula, the S-parameter obtained by Matlab simulation is shown in Figure 1, where the coupling coefficient of each branch, external $Q$ value and other initial values are shown in Table 2. Each a separate filter circuit is Bandpass filter with transmission zeros equivalent circuit, the two channel filter through a public space together, after the synthesis of duplexers topology structure as shown in figure 2 , the hollow circle represents the source and load, solid lines represent the main coupling, dashed line represents the cross coupling.

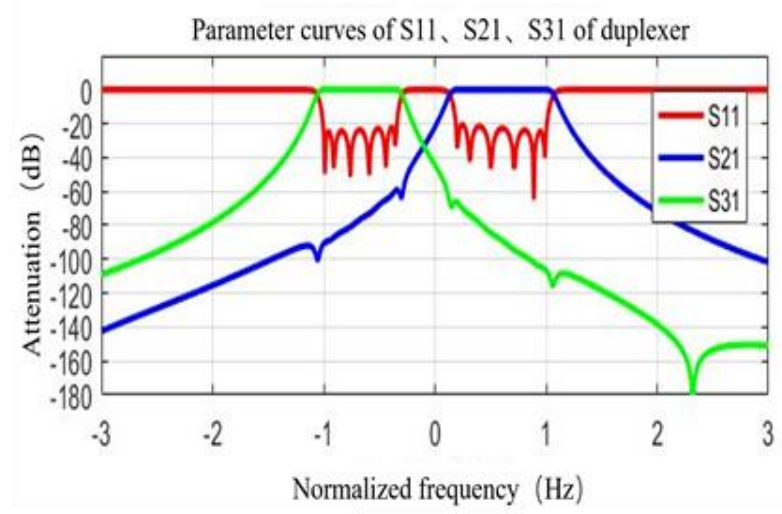

Figure 1 Matlab simulation of S parameters

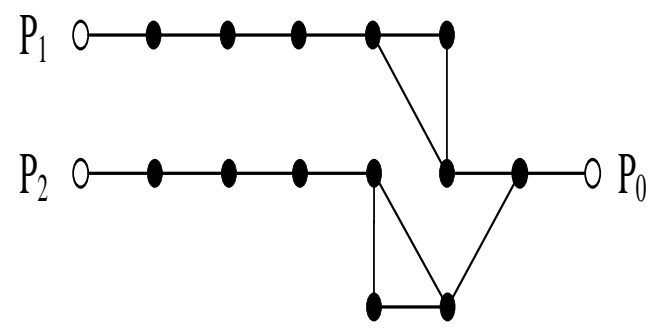

Figure 2 Topology diagram of combiner

Table 2 The coupling coefficient and the external $Q$ value of the duplex

\begin{tabular}{ccc}
\hline$K$ & Band 1 & Band 2 \\
\hline$K 12$ & 0.0425 & 0.0393 \\
$K 23$ & 0.0147 & 0.0123 \\
\hline
\end{tabular}

\begin{tabular}{ccc}
\hline K34 & 0.0119 & 0.0097 \\
$K 45$ & 0.0114 & 0.0093 \\
$K 56$ & 0.0121 & 0.0099 \\
$K 67$ & 0.0171 & 0.0141 \\
$K 13$ & 0.0009 & -0.0009 \\
$Q_{e}$ & 46.6643 & 55.6215 \\
Common port $Q_{e}$ & & 9.0668 \\
\hline
\end{tabular}

Figure 2 shows that the order of the band-pass filter with a Passband frequency of $1550-1580 \mathrm{MHz}$ is 7 , and that of the band-pass filter with a Passband frequency of $1600-1625 \mathrm{MHz}$ is 7 . In the design, the bandpass filter adopts cross coupling structure, and the circuit model of duplexer established by the topology structure in Figure 2 is shown in Figure 3 by using the circuit simulation design software ADS.

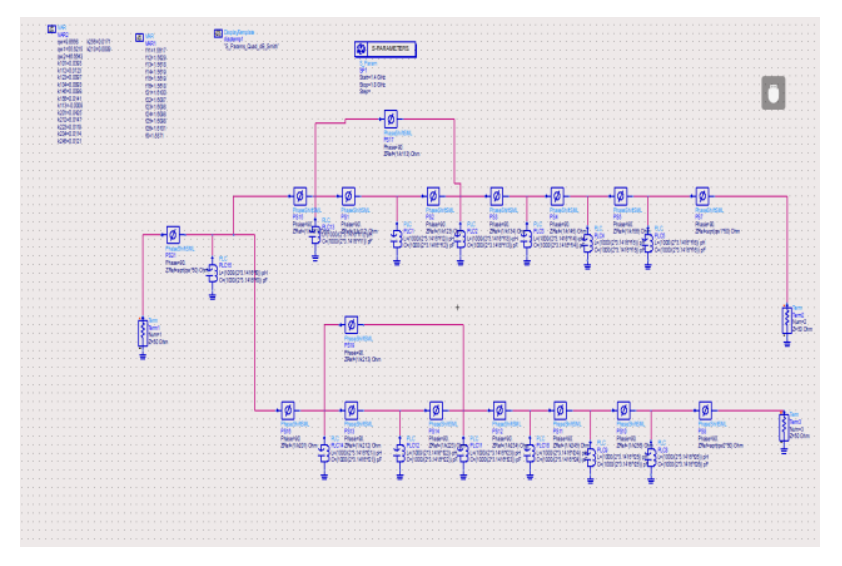

Figure 3 Circuit model of combiner

\section{DUPLEX MODEL SIMULATION}

HFSS software was used to simulate the combiner, and according to the optimized design parameters extracted from the ADS circuit model, the common end coupling structure, the coupling dimensions of each input end and the tap position were calculated. The structure of a single cavity can be determined by the resonant frequency through single cavity simulation by HFSS. For the extraction of the coupling coefficient between resonators, the eigen mode of HFSS is needed. In the solution environment of the eigen mode, the size of the coupling coefficient can be obtained, and the formula is as follows:

$$
K=\frac{f_{m}^{2}-f_{e}^{2}}{f_{m}^{2}+f_{e}^{2}}
$$

Where $f_{m}$ and $f_{e}$ are two single cavity resonant frequencies.

The coupling modes of tap structure include direct coupling, capacitive coupling and inductive coupling. The dimension of the multiplexer is determined by simulation of variables sensitive to the influence of circuit parameters, in which the resonant frequency is determined by the depth of the frequency modulation screw $d$, the coupling coefficient between adjacent cavities is determined by the width of the side wall window $w$, and the on-load $Q$ value is determined by the height of the solder joint position $H$. Then select appropriate scanning interval and step length to scan each parameter one by one, extract the circuit parameters in the 3D model, and establish the quantitative relationship between the circuit parameters and the corresponding model size. Finally, the optimized model size 
parameters in HFSS are shown in Table 3.The simulation model is shown in Figure 4.

Table 3 Duplex dimension Table (unit: $\mathrm{mm}$ )

\begin{tabular}{|c|c|c|c|}
\hline \multicolumn{2}{|c|}{ GPS channel } & \multicolumn{2}{|c|}{ Lshort message channel } \\
\hline \multicolumn{4}{|c|}{$\begin{array}{l}\text { Frequency modulation screw coupling window width and solder } \\
\text { joint position }\end{array}$} \\
\hline $\mathrm{d} 1=1.8$ & $w 12=7.6$ & $\mathrm{~d} 1=2.1$ & $\mathrm{w} 12=7.3$ \\
\hline $\mathrm{d} 2=2.0$ & $\mathrm{w} 23=6.4$ & $\mathrm{~d} 2=1.6$ & $\mathrm{w} 23=6.3$ \\
\hline $\mathrm{d} 3=1.5$ & w34 = 6.2 & $\mathrm{~d} 3=1.3$ & $\mathrm{w} 34=6.2$ \\
\hline $\mathrm{d} 4=1.5$ & $\mathrm{w} 45=6.5$ & $\mathrm{~d} 4=1.2$ & $\mathrm{w} 45=6.3$ \\
\hline $\mathrm{d} 5=2.0$ & w56 $=7.0$ & $\mathrm{~d} 5=1.3$ & $\mathrm{w} 56=6.8$ \\
\hline $\mathrm{d} 6=1.8$ & & $\mathrm{~d} 6=1.8$ & \\
\hline $\mathrm{H}=8.0$ & & $\mathrm{H}=8.0$ & \\
\hline
\end{tabular}

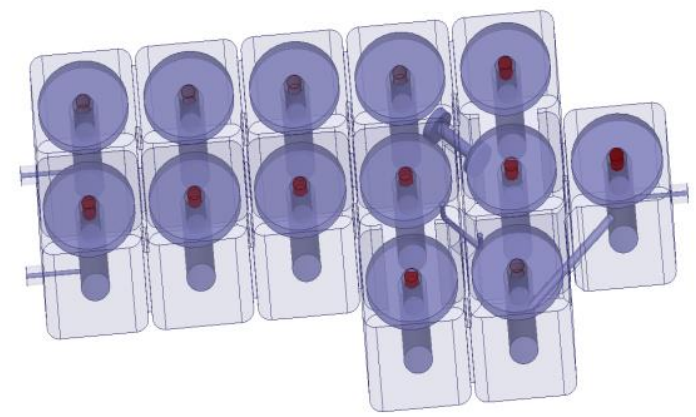

Figure 4 The simulation model of combiner

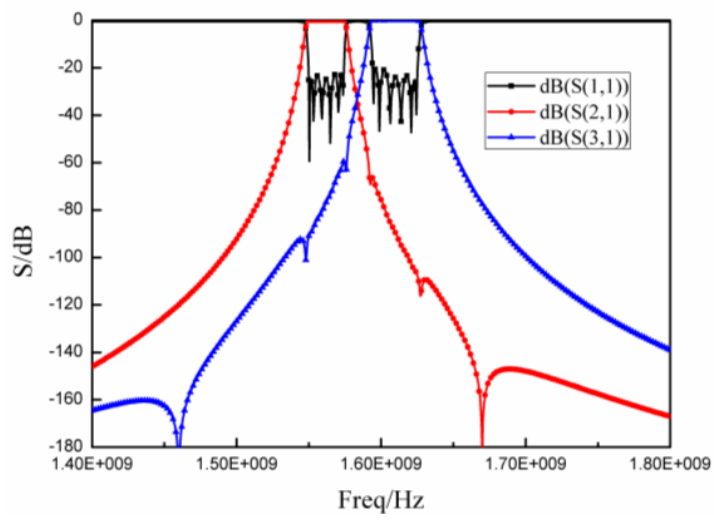

Figure 5 The optimization results of combiner

The overall model of the duplex as shown in Figure 4 is simulated, and the final result is shown in Figure 5.

Figure 5 shows that the simulation return loss in the frequency band $1550-1580 \mathrm{MHz}$ is less than $-20 \mathrm{~dB}$, the insertion loss is less than $0.5 \mathrm{~dB}$, and the return loss in the frequency band 1600 $1625 \mathrm{MHz}$ is less than $-20 \mathrm{~dB}$, and the insertion loss is less than $0.5 \mathrm{~dB}$, which is consistent with the expected results and proves the previous design theory.

\section{CONCLUSION}

In this thesis, we design a miniaturized dual combiner, based on the basic theory of filter Matlab the coupling coefficients of draw two line filter parameters, such as using circuit simulation software ANSOFT DESIGNER for circuit simulation and optimization, and through the simulation software HFSS design meets all design index, a simulation model of each filter all the way to make good, restraint outside the band can improve the isolation. The design efficiency is high, the engineering practicality is strong, can satisfy the modern communication system to combiner miniaturization, the high isolation degree request.

\section{ACKNOWLEDGMENTS}

First of all, I would like to thank my research tutor for his guidance, who pointed out the problems existing in the thesis for me. Secondly, Yang Huan helped me to successfully complete the measurement of the experimental results. I really thank them for helping me to finish this paper successfully.

\section{REFERENCES}

[1] Macchiarella G, Tamiazzo S. Synthesis of Star-Junction Multiplex-ers [J]. IEEE Transactions on Microwave Theory and Techniques, 2010, 58(12), 3732-3741.

[2] Kim B, Kim Y S. Mixed Coupling Structure for the Cross Couplingof Combline Filters [J]. Microwave and Optical Technology Let-ters, 2002, 35 (1) : 20-23.

[3] Hong J S, Lancaster M J. Couplings of Microstrip Square Open-Loop Resonators for Cross-Coupled Planar Microwave Filter[J]. IEEE Transactions on Microwave Theory and Techniques, 1996, 44 (11) : 2099-2109.

[4] Feng Jianhua. Miniaturization design of multifrequency coaxial cavity combiner [J]. Mobile Communication, 2016,40(19):60-65.

[5] Chen Qihao, Ye Qiang, Feng JianHua. Design of a highisolation cavity dual-frequency comb-solver [J]. Electronics,2016,39(02):276-279.

[6] Cameron R J. General Coupling Matrix Synthesis Methods for Chebyshev Filtering Functions [J]. Microwave Theory and Techniques, IEEE Transactions on, $1999,47(4): 433-442$.

[7] Chen Qihao, YE Qiang, Feudal Hua. Design of a highisolation cavity dual-frequency combine $[\mathrm{J}]$. Journal of Electron Device, 2016, 39(02): 276-279. 